\title{
ESTABLECIMIENTO DE PLANTAS MEDIANTE EL USO DE MICORRIZAS Y DE ISLAS DE RECURSOS EN UN MATORRAL XERÓFILO DETERIORADO
}

\author{
Arcadio Monroy-Ata', Juan Estevez-Torres, Rosalva García-Sánchez \\ Y RAMIRO RÍOS-GÓMEZ
}

Unidad de Investigación en Ecología Vegetal, Carrera de Biología, FES-Zaragoza, Universidad Nacional Autónoma de México, Apdo. Postal 9-020, México 15000, D.F., México.

'Autor para la correspondencia: Tel. y Fax: 52 (55) 5623-0768; correo-e: arcadiom@servidor.unam.mx

\begin{abstract}
Resumen: En este trabajo se evaluó la supervivencia de plantas de mezquite (Prosopis laevigata) y de huizache (Acacia farne siana), previamente inoculadas con hongos micorrizógenos arbusculares (HMA), en condiciones de campo, durante un año. Las plantas fueron cultivadas durante nueve meses en invernadero y dos meses en vivero, antes de ser trasplantadas a un matorral xerófilo deteriorado localizado en el Valle de Actopan, Hidalgo, México. Cada individuo, micorrizado o no micorrizado, fue transplantado bajo la cobertura de una planta nodriza perteneciente a una de ocho especies generadoras de islas de recursos, con el fin de evaluar el efecto de la micorrización y del nodrizaje vegetal sobre el establecimiento y la supervivencia de las plantas, durante el periodo de sequía (octubre-abril). Los resultados muestran que la micorrización de plantas de mezquite y de huizache aumenta de manera significativa la supervivencia, incrementándose el porcentaje de 19 a 54\% para P. laevigata, y de 18 a $48 \%$ para A. farnesiana. Respecto a la especie de planta nodriza, no se encontraron diferencias en el porcentaje de supervivencia de los individuos trasplantados debidas al tipo de isla de recursos. El experimento también mostró que las plantas micorrizadas desarrollan mayor número de pinnas con relación a sus testigos. Se concluye que es recomendable la inoculación de plántulas con HMA nativos, en programas de restauración ecológica de ecosistemas semiáridos deteriorados.
\end{abstract}

Palabras clave: Acacia farnesiana, establecimiento vegetal, hongos micorrizógenos arbusculares, Prosopis laevigata.

\begin{abstract}
Survival of mesquite (Prosopis laevigata) and huizache (Acacia farnesiana), previously inoculated with arbuscular mycorrhizal fungi (AMF) was analyzed in field conditions during one year. The plants were cultivated for nine months in a greenhouse, two months in a nursery, and then they were transplanted in a deteriorated xeric shrub ecosystem located in Actopan Valley, Hidalgo State, Mexico. Each individual (either inoculated or not inoculated with AMF), was transplanted under the cover of one of eight nurse plant species forming resource islands, in order to evaluate the effects of mycorrhizal inoculum and nurse plant identity on plant establishment and survival during the dry period (October to April). Overall, results show that inoculated mesquite and huizache plants had a significant increase in percent survival, raising from 19 to $54 \%$ for P. laevigata, and from 18 to $48 \%$ for A. farnesiana. Regarding nurse plant identity, i.e. resource island type, there were not significant differences in percent survival of transplanted individuals. The experiment also showed that inoculated plants had a larger number of pinnae than their controls. On this basis, inoculation of seedlings with AMF is recommended to restore deteriorated semi-arid ecosystems. Key words: Acacia farnesiana, arbuscular mycorrhizal fungi, plant establishment, Prosopis laevigata.
\end{abstract}

L as zonas áridas y semiáridas de México ocupan entre $50 \%$ y $60 \%$ de la superficie total del país (Challenger, 1998). Estas regiones de baja precipitación pluvial anual son referidas usualmente como áridas, si su precipitación media anual es inferior a $250 \mathrm{~mm}$, y como semiáridas si la lluvia media fluctúa entre 250 y 450 mm (Nobel, 1998). En estos ambientes, el clima y la topografía son los factores que determinan, en mayor medida, los patrones de distribución espacial y temporal de la vegetación en las comunidades vegetales (Valentin et al., 1999).

Los ecosistemas áridos y semiáridos en México poseen una elevada diversidad, de alrededor de 6,000 especies, y un porcentaje importante de endemismos: $65 \%$ de los géneros y ca. $60 \%$ de las especies que medran en estos 
ambientes (Rzedowski, 1991). Sin embargo, las zonas áridas y semiáridas mexicanas presentan señales de deterioro en amplios temitorios del país, debido a actividades humanas como el sobrepastoreo, la extracción de leña, la sobreexplotación de algunas especies y los incendios. Por ello, es necesario buscar técnicas que permitan revertir la pérdida de vegetación y la erosión del suelo.

Una zona que ejemplifica esta situación es el Valle del Mezquital en el estado de Hidalgo (centro de México), donde los recursos bióticos han sido utilizados continuamente desde la época prehispánica por grupos de la etnia otomí (ñhañhú). Una de las unidades fisiográficas que conforman esta región semidesértica es el Valle de Actopan, en el cual se encuentran diversos tipos de vegetación, siendo el matorral xerófilo el más común. En estos ecosistemas coexisten especies leñosas de las familias Fabaceae, Agavaceae, Cactaceae y Liliaceae (yucas), con hierbas como gramíneas (Poaceae) y compuestas (Asteraceae). El nombre de la región se debe al mezquite [Prosopis laeviga ta (Humb. et Bonpl. ex Willd.) M.C.Johnst.]; esta especie ha sido particularmente sobreexplotada por ser una planta de usos múltiples (madera, forraje, alimento, resina, etc.; Galindo-Almanza y García-Moya, 1986). Otra especie importante en la economía de los pobladores rurales de esta región es el huizache o acacia [Acacia farnesiana (L.) Willd.] (Fagg y Stewart, 1994), el cual es utilizado como fuente de leña, carbón, madera y forraje. Las dos especies pertenecen a la familia de las leguminosas, $\mathrm{c}$ recen en forma arbustiva o arbórea y son importantes desde el punto de vista ecológico, ya que son plantas caducifolias que periódicamente aportan materia orgánica al suelo bajo su cobertura. Además, ambas especies son fijadoras de nitrógeno atmosférico a través de una simbiosis, por lo que se les ha llamado especies formadoras de "islas de fertilidad" (GarcíaM oya y McKell, 1970; Cuz z, 1992, 1996). Más recientemente se ha propuesto el concepto de "islas de recursos" en vez de "islas de fertilidad" (Camarg-Ricalde y Dhillion, 2003). Sin embargo, las leguminosas arbustivas y arbóreas como los mezquites, los huizaches y las mimosas (Mimosa spp.) no sólo contri bu yen al incremento de nu trientes bajo su cobertura, sino que además promueven la producción de propágulos micorrícicos, crean un microclima favorable para el establecimiento de otras plantas, condensan neblina en los inviernos fríos y secos del Altiplano Mexicano (aportando humedad adicional al suelo fuera del periodo de lluvias), y protegen de heladas y de precipitaciones pluviales intensas a las plantas que crecen bajo su dosel.

A la planta leñosa que genera una isla de recursos se le ha denominado planta nodriza, y se considera que se establece una relación de protocooperación entre ésta y el conjunto de especies vegetales que crecen bajo su cobert ura, ya que las plantas asociadas a la especie nucleadora comparten la presión de herbivoría, contribu yen al mantenimiento del micro clima mediante su transpiración y aportan materia orgánica a la isla de recursos (Callaway, 1995). El nodrizaje es un proceso que ha sido explicado en función de la sombra que genera la planta nucleadora (Valiente-Banuet y Ezcurra, 1991), el aumento en la retención de humedad (Franco y Nobel, 1989), el incremento en la fertilidad del suelo (Garner y Steinberger,1989), y la generación de propágulos micorrícicos (Camargo-Ricalde y Dhillion, 2003). Asimismo, se denomina micrositio de establecimiento vegetal al lugar que reúne las condiciones favorables para el desarrollo de una plántula, desde su emergencia hasta completar un ciclo anual (Maestre et al., 2002a).

Otrainteracción biótica importante en el establecimiento vegetal consiste en un proceso dinámico, que integra funcionalmente plantas y hongos micorrizógenos, mediante una asociación mutualista conocida como micorriza. Esta relación simbiótica es cosmopolita y ha sido observada en una amplia variedad de ecosistemas naturales y de campos de cultivo. La abundancia de las micorrizas en los ecosistemas terrestres se debe a que éstas permiten una absorción más efectiva de agua y nutrientes, especialmente fosfatos, para las plantas (Allen y Allen, 1992).

En el caso de las zonas áridas y semiáridas, el factor más limitante del desarrollo vegetal es la disponibilidad de agua (Wiegand et al., 1999), además de que los suelos de estos ambientes son generalmente pobres en fósforo. Por esta razón, el papel de los hongos micorrizógenos arbusculares (HMA) es determinante en el crecimiento en estos ecosistemas.

Diversos estudios han reportado que la aplicación de inóculos de HMA es una alternativa recomendable para favorecer el establecimiento y crecimiento de plantas, en programas de recolonización vegetal de suelos (Barea, 1998; Camargo-Ricalde, 2001). En consecuencia, la rehabilitación de la vegetación de ecosistemas deteriorados puede inducirse, con mayores probabilidades de éxito, si se emplean plantas micorrizadas con inóculos nativos.

En este trabajo se decidió evaluar el establecimiento de plantas de mezquite (Prosopis laevigata) y de huizache (Acacia farmesiana), inoculando plántulas con hongos micorrizógenos en condiciones de invernadero, aclimatando los individuos en un vivero y trasplantándolos bajo la cobertura de una planta nodriza. Este procedimiento no sólo tuvo como fin de rehabilitar la vegetación, sino que también se hizo para recuperar la biota edáfica de un ecosistema deteriorado, como ha sido propuesto por Azcón y Barea (1997) y Azcón-Aguilar et al. (2003). La pregunta a responder con este trabajo fue: ¿la micorrización de plántulas de mezquite y de huizache incrementa el porcentaje de supervivencia, en condiciones de campo, después de un ciclo anual? 


\section{Materiales y métodos}

Zona de estudio. Es un matorral xerófilo ubicado al NO del poblado de Santiago de Anaya $\left(20^{\circ} 22\right.$ '59" 1 atitud N; 98 58'06" longitud O), perteneciente al municipio del mismo nombre y localizado en el Valle de Actopan (estado de Hidalgo, México), a $15 \mathrm{~km}$ al norte de la ciudad de Actopan. La altitud de la zona de estudio es de $2,024 \mathrm{~m}$ s.n.m. El clima es templado con régimen de lluvias en ve rano. La temperatura media anual del sitio está comprendida entre 16 y $20^{\circ} \mathrm{C}$, con $550 \mathrm{~mm}$ de precipitación media anual. El tipo de vegetación es matorral subinerme con dominancia de Flourensia resinosa y Mimosa depauperata. Es una zona semiárida donde las principales causas de deterioro son el sobrepastoreo y la destrucción de la vegetación por desmonte para fines agrícolas, y donde actualmente se observan signos de erosión, lo que ocasiona la falta de retención de agua por ser suelos someros y la presencia de afloramientos de tepetate.

Especies. Se recolectaron frutos maduros (legumbres) de mezquite [Prosopis laevigata (Humb. et Bonpl. ex Willd.) M.C.Johnst. (Fabaceae)] y de huizache [Acacia farnesiana (L.) Willd. (Fabaceae)], en el Valle del Mezquital, estado de Hidalgo, México. Las semillas fueron separadas y escarificadas mediante incisión en la testa y se pusieron a germinar en suelo estéril, en 140 macetas por especie. En cada maceta se colocaron dos semillas y cuando ambas germinaron y emergieron, después de una semana, se eliminó una de las plántulas.

Inóculo micorrícico. Se recolectó suelo en un mat o rral xerófilo poco perturbado, dominado por Mimosa biuncifera Benth. en el estrato arbustivo y por gramíneas [Bouteloua gracilis (Kunth) Lag. ex Griffiths y B. curtipendula (Michx.) To rr. in Marcy] en el estrato herbáceo. La recolecta se realizó en la época seca, tomando las mu e s tras de suelo entre 0 y $20 \mathrm{~cm}$ de profundidad, en sitios donde crecían macollos del zacate navajita azul (B. gracilis). La masificación de los HMA se realizó en condiciones de invernadero, se mb rando semillas de una planta "trampa", en este caso del pasto anual "rye grass" (Lolium multiflorum), en macetas con un sustrato compuesto por suelo (proveniente de un matorral xerófilo del campo ex p e rimental en el Valle de Actopan) y arena sílica, en proporción 1:1 (v/v). Este sust rato fue esterilizado mediante calor húmedo en dos ocasiones en días consecutivos. Adicionalmente, encima de este sustrato se colocó una capa de $1 \mathrm{~cm}$ de espesor del suelo seco con esporas de HMA, recolectado en el ecosistema poco deteri o rado. Después de seis meses se disminuyó paulatinamente el riego de la planta trampa para fomentar la esporulación de los hongos micorrizógenos; una vez que se marchitaron las plantas del pasto anual, se retiró la parte aérea y se hizo el conteo de esporas de hongos mico- rri zó genos por cien gramos de suelo. Este sustrato, con las raíces induidas, se utilizó como inóculo micorrícico.

Fase de invernadero. El invemadero está ubicado en el o riente de la ciudad de México, Distrito Fe deral; está orientado de norte a sur y fue utilizado con las ventanas abiertas. En macetas cilíndricas, con un diámetro de $0.075 \mathrm{~m}$ y una altura de $0.30 \mathrm{~m}$, se colocó una mezcla de suelo (proveniente del campo experimental en el Valle de Actopan) con grano de mármol (1:1), esterilizada previamente en autoclave a 15 libras de presión durante $2 \mathrm{~h}$ al día, por tres días consecutivos; después, se puso una capa de 100 g de inóculo micorrícico con una densidad de esporas de 1,500/100 g de suelo seco; una vez que quedó seleccionada una plántula en cada una de las macetas, éstas fueron regadas semanalmente, con agua destilada a capacidad de campo.

Diseño experimental. Se cultivaron 140 plantas por especie (mezquite y huizache), 60 plántulas con inóculo micomícico y 60 testigos, más un lote de 20 macetas (10 micomizadas y 10 no micorrizadas), para evaluar la colonización micorrícica después de su crecimiento en inver$\mathrm{n}$ a de roy vive ro y antes de ser trasplantadas a campo. En el invemadero se midieron como variables de respuesta la alturay el número de pinnas; en ambas especies se registró el número de pinnas, ya que las hojas están compuestas por dos a ocho pares de pinnas, por lo que éstas son un mejor indicador del desarrollo foliar.

Fase de vivero. Después de un periodo de nueve meses de cultivo de las plantas en invernadero, y con el fin de favorecer el transplante a condiciones de campo, las macetas fueron llevadas a un vivero (localizado en el campus de la Facultad de Estudios Superiores Zaragoza de la Universidad Nacional Autónoma de México), donde permanecieron dos meses con riegos semanales antes de ser llevadas a campo.

Evaluación de la colonización micorrícica. Al final del periodo de invernadero y de vivero, se seleccionó al azar un lote de 20 plantas de cada especie (10 micorrizadas y 10 no micorrizadas), el cual fue utilizado para determinar el porcentaje de colonización micorrícica. Para esto, las raíces se fijaron en alcohol etílico al 50\%. La colonización micorrícica fue evaluada por el método de tinción con azul de tripano, de acuerdo con la técnica propuesta por Phillips y Hayman (1970).

Transplante a campo. En campo se trasplantaron 117 individuos de mezquite y 116 de huizache, los cuales se ubicaron bajo la cobertura -y al lado norte- de una planta adulta, de una de las ocho especies seleccionadas como nodriza. Las especies utilizadas como nodriza fueron plantas leñosas dominantes y codominantes en un matorral 
xerófilo deteriorado por sobrepastoreo y fueron las siguientes: Agave salmiana Otto (Agavaceae), Condalia mexicana Schltdl. (Rhamnaceae), Flourensia resinosa S.F.Blake (Asteraceae), Karwinskia humboldtiana S.Watson (Rhamnaceae), Mimosa depauperata Benth. (Fabaceae), Opuntia cantabrigiensis Lynch (Cactaceae), Opuntia imbri cata (Haw.) DC. (Cactaceae) y Prosopis laevigata (Humb. et Bonpl. ex Willd.) M.C.Johnst. (Fabaceae). En cada planta nodriza se trasplantó un individuo de mezquite o de huizache, como se muestra en el cuadro 1.

El diseño experimental fue un factorial de 2 especies $\times 2$ tratamientos $\times 8$ nodrizas $\times 4$ a 14 repeticiones. El análisis de datos se realizó para cada especie (mezquite y huizache) con un ANDEVA de dos factores: micorrización (con dos niveles) $\times$ especie de planta nodriza utilizada (ocho especies). La evaluación del porcentaje de supervivencia en la última fe cha de registro, para ambas especies, se realizó mediante una comparación de medias (prueba de $t$ ) entre plantas micorrizadas y no micorrizadas. En todas las plantas se registraron mensualmente y durante 12 meses las siguientes variables: porcentaje de supervivencia, altura y número de pinnas.

\section{Resultados}

Colonización micorrícica. El cuadro 2 muestra los resultados de la evaluación de la colonización micorrícica de las plantas de mezquite y de huizache, a los once meses de edad y antes del trasplante a un matorral xerófilo deteriorado; el porcentaje promedio de colonización total supera $50 \%$ en las dos leguminosas. Asimismo, en ambas especies se detectaron arbúsculos, lo que permite verificar que el

Cuadro 1. Número de réplicas de individuos de Prosopis laevigata (mezquite) y de Acacia farnesiana (huizache), micorrizados (M+) y no micorrizados (M-), trasplantados bajo la copa de una planta de una de las especies nodriza.

\begin{tabular}{lcccccc}
\hline Especie de nodriza & \multicolumn{2}{c}{ Prosopis laevigata } & & \multicolumn{2}{c}{ Acacia farnesiana } \\
\cline { 2 - 3 } \cline { 6 - 7 } & M+ & M- & & M+ & M- \\
\hline Agave salmiana & 5 & 5 & & 6 & 6 \\
Condalia mexicana & 6 & 5 & & 4 & 6 \\
Flourensia resinosa & 10 & 13 & & 13 & 12 \\
Karwinskia humboldtiana & 6 & 5 & & 7 & 6 \\
Mimosa depauperata & 14 & 12 & & 9 & 13 \\
Opuntia cantabrigiensis & 5 & 6 & & 5 & 4 \\
O. imbricata & 6 & 7 & & 7 & 6 \\
Prosopis laevigata & 6 & 6 & & 6 & 6 \\
Total & 58 & 59 & & 57 & 59 \\
\hline
\end{tabular}

inóculo es funcional y que contenía inicialmente propágulos viables de HMA.

Porcentaje de supervivencia. La supervivencia de las plantas al final de un ciclo anual, independientemente del tipo de nodriza, fue mayor para las plantas micorrizadas en ambas especies, ya que el porcentaje de supervivencia de las plantas inoculadas con HMA fue 2.8 veces superior a los testigos, mostrando diferencias estadísticamente significativas $(P=0.027$ en $P$. laevigata y $P=0.04$ en $A$. farne siana; figura 1a, b). En los dos casos, las curvas muestran una mortalidad inicial baja hasta el día 200; a partir de esta fecha la mortalidad se incrementó coincidiendo con la época más seca del año, con un comportamiento similar entre tratamientos, aunque la curva de las plantas micomizadas presenta valores de supervivencia significativamente más elevados.

Cuadro 2. Porcentajes promedio de colonización micorrícica de las plantas inoculadas con hongos micorrizógenos arbusculares.

\begin{tabular}{lcc}
\hline Tipo de colonización & Prosopis laevigata & Acacia farnesiana \\
\hline Vesículas & 35.29 & 35.85 \\
Arbúsculos & 12.11 & 13.00 \\
Total & 53.86 & 54.10 \\
\hline
\end{tabular}
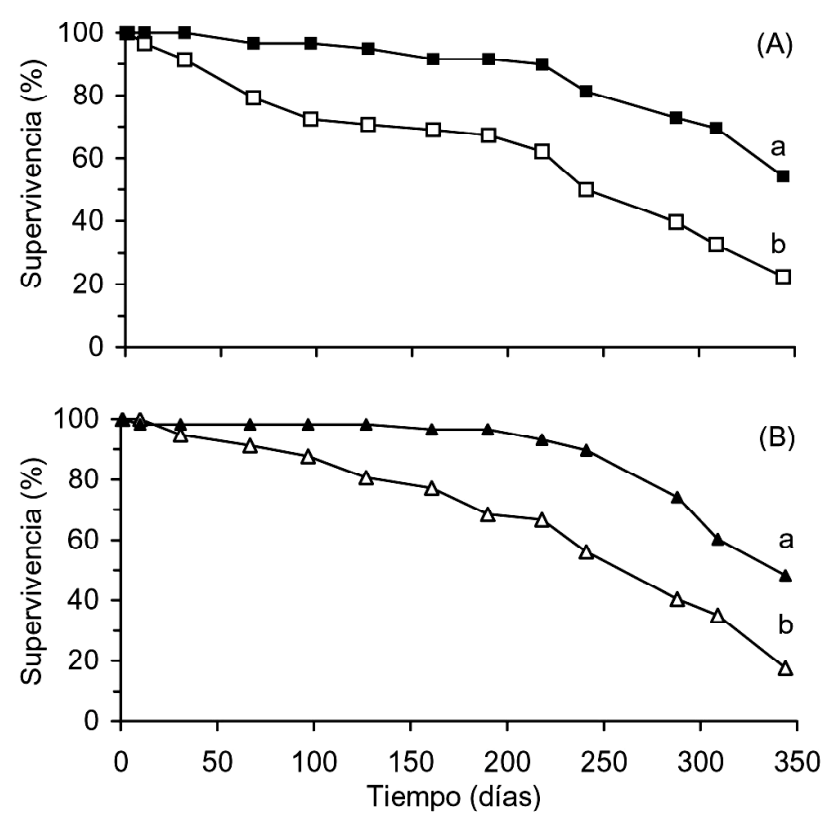

Figura 1. Supervivencia de Prosopis laevigata (A) y de Acacia farnesiana (B) en campo. Letras minúsculas distintas indican diferencias significativas $(P<0.05)$ en la supervivencia final. Plantas micorrizadas (símbolos llenos); plantas no micorrizadas (símbolos vacíos). 
Islas de recursos. Las curvas de supervivencia para Prosopis laevigata bajo las ocho especies de plantas nodrizas muestran comportamientos diferenciales, entre los que destacan, para el caso de las plantas micorrizadas, las que crecen bajo Agave salmiana por presentar una alta mortalidad de plántulas de mezquite desde el inicio del ciclo, siendo al final del periodo anual la nodriza bajo la cual se presenta la mayor mortalidad (80\%). Condalia mexicana mantuvo la superv ivencia de plantas de mezquite c e rano a $100 \%$ hasta el día 300 del ciclo; sólo en la fase final, que corresponde a la época más seca del año, presentó una elevada mortalidad, llegando a $60 \%$ de supervivencia. La nodriza bajo la cual se presentó el más alto porcentaje de supervivencia de plantas de mezquite al final del registro fue Karwinskia humboldtiana, con $80 \%$ (figura 2a).

En el caso de las plantas no micorrizadas, destaca Mimosa depauperata como la nodriza bajo la cual se pre- sentó la mayor mortalidad desde el inicio del ciclo, para finalizar con una supervivencia de tan sólo $14.3 \%$. Por el contrario, Condalia mexicana, junto con Flourensia resinosa y Karwinskia humboldtiana, fueron las nodrizas que favorecieron la supervivencia más alta $(50 \%)$ de las plantas de mezquite. En las otras nodrizas (Agave salmiana, Opuntia cantabrigiensis, O. imbricata y Prosopis laeviga ta) se presentó una supervivencia de los individuos trasplantados de $80 \%$ hasta el día 200; a partir de este momento la mortalidad de mezquite fue alta, llegando a $100 \%$ en el caso de Opuntia cantabrigiensis (figura 2b).

En el caso de las plantas micorrizadas de Acacia farne siana, se observó un comportamiento similar bajo las ocho nodrizas, excepto para Agave salmiana, ya que en esta nodriza se presentó una alta mortalidad de los individuos trasplantados (ca. 20\%) desde la primera fecha; sin embar-
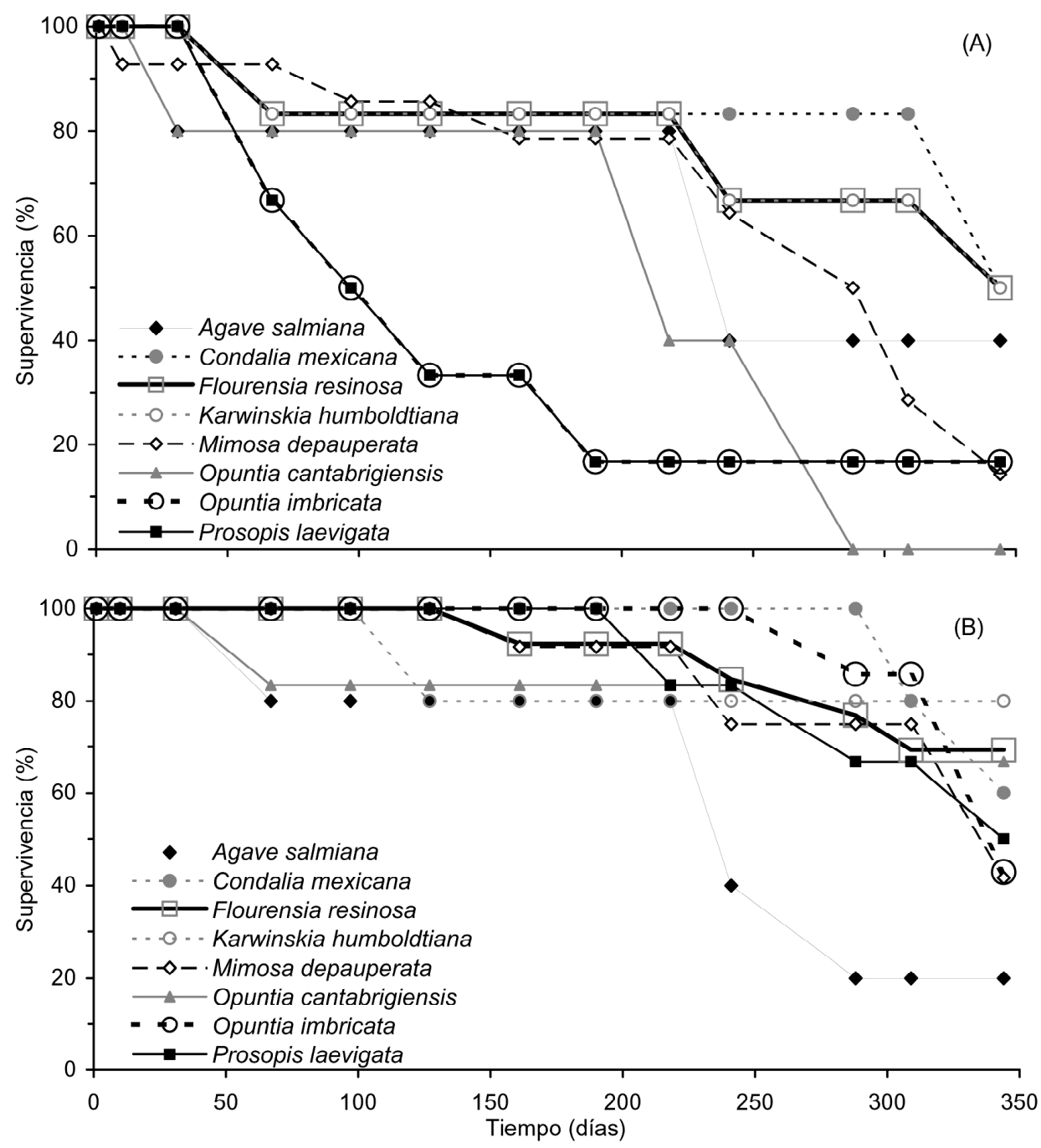

Figura 2. Supervivencia de plantas de Prosopis laevigata micorrizadas (A) y no micorrizadas (B), bajo la cobertura de ocho especies de plantas nodriza. 
go, la supervivencia al final del periodo de evaluación fue de $66 \%$. A partir del día 250 , la mortalidad de plantas fue notable, llegando a $100 \%$ en un corto período, como en el caso de Condalia mexicana. Mimosa depauperata destacó como especie de nodriza por haber favorecido la mayor supervivencia de plantas de huizache, con valores de $76.9 \%$ (figura 3a).

En el caso de las plantas no micorrizadas de Acacia far nesiana, bajo las ocho especies de nodriza el porcentaje de supervivencia fue similar entre ellas, excepto en el caso de Condalia mexicana, la cual mantuvo una mayor supervivencia inicial hasta el día 200, para después mostrar una caída hasta llegar al final a 100\% de mortalidad, al igual que en el caso de las nodrizas Karwinskia humboldiana y Opuntia cantabrigiensis (figura 3b).

Establecimiento vegetal en campo. En la figura 4a se muestra la altura promedio de las plantas de Prosopis laevigata; en este caso se observó al inicio un decremento en la talla de las plantas micorrizadas y al final del ciclo anual tuvieron la misma altura que las plantas no micorrizadas, las cuales mantuvieron su altura promedio inicial. En el registro del número de pinnas promedio por planta (figura 4b), se observó que las plantas micorrizadas tuvieron una defoliación inicial; después, en el periodo de lluvias, recuperaron el número de pinnas promedio y posteriormente se redujeron a cero en la época seca, lo cual corresponde a una especie caducifolia. Al final del ciclo anual, la gráfica presentó un aumento en el número de pinnas, en el caso de las plantas micorrizadas, mientras que en el testigo el comportamiento fue similar, pero el número de pinnas fue menor (figura 4b).

Respecto a Acacia farnesiana, la variación en altura durante el ciclo anual de las plantas micorrizadas y no micorrizadas tuvo un comportamiento similar, pero las plantas micorrizadas siempre fueron más altas (figura 5a).
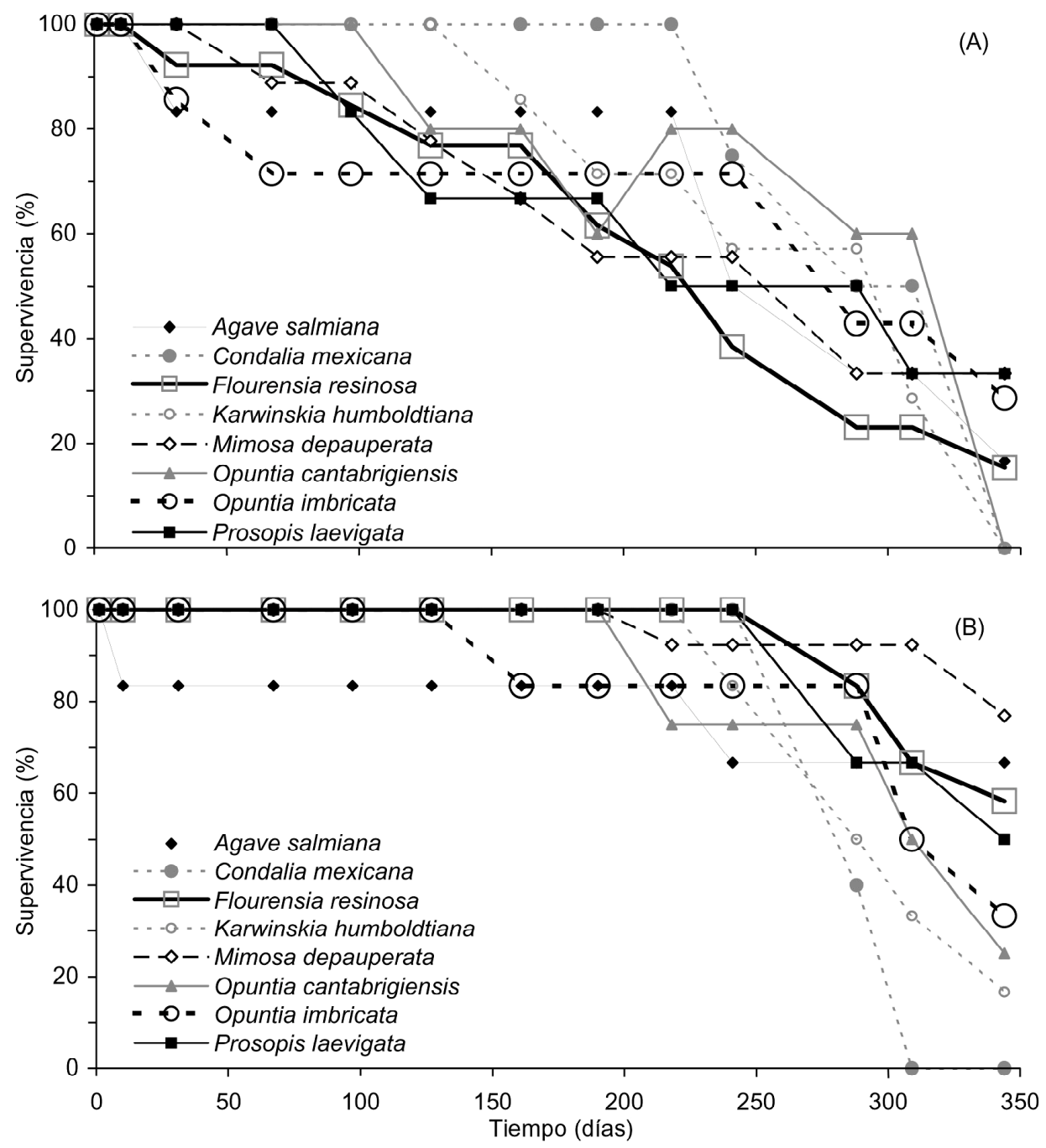

Figura 3. Supervivencia de plantas de Acacia farnesiana micorrizadas (A) y no micorrizadas (B), bajo la cobertura de ocho especies de plantas nodriza. 
En cuanto al número de pinnas promedio en las plantas micorrizadas de Acacia, se observó un ligero decremento inicial para inmediatamente aumentar, incluso por arriba del número inicial; después, con el avance del ciclo anual se observó una pérdida de pinnas, lo cual se debe a la época de secas del año, dado que es una especie caducifolia. Las plántulas no micorrizadas presentaron un comportamiento similar, coincidiendo únicamente cuando el número de las pinnas fue cero. Al final del ciclo se observó un aumento en el número de pinnas tanto en las plantas micorrizadas como en sus testigos; sin embargo, el aumento fue mayor en el caso de las plantas inoculadas con HMA (figura $5 b$ ).

\section{Discusión}

Colonización micorrícica. La masificación de esporas de hongos micorri zó genos arbusculares, provenientes del suelo que rodea las raíces del zacate navajita azul (Boutelona gra cilis) resultó ser un inóculo micorrícico con alto grado de colonización de raíces ( $>50 \%)$. Una caracterzación preliminar de este inóculo mostró una dominancia de esporas del género Glomus. Al respecto, Allen et al. (2003) mostraron que un inóculo proveniente de las primeras etapas serales de un bosque tropical, con una mayoría de esporas de Glomus, favo reció el crecimiento de especies arbóreas tanto de etapas serales tempranas como tardías; en contraste, inóculos provenientes de etapas serales maduras,
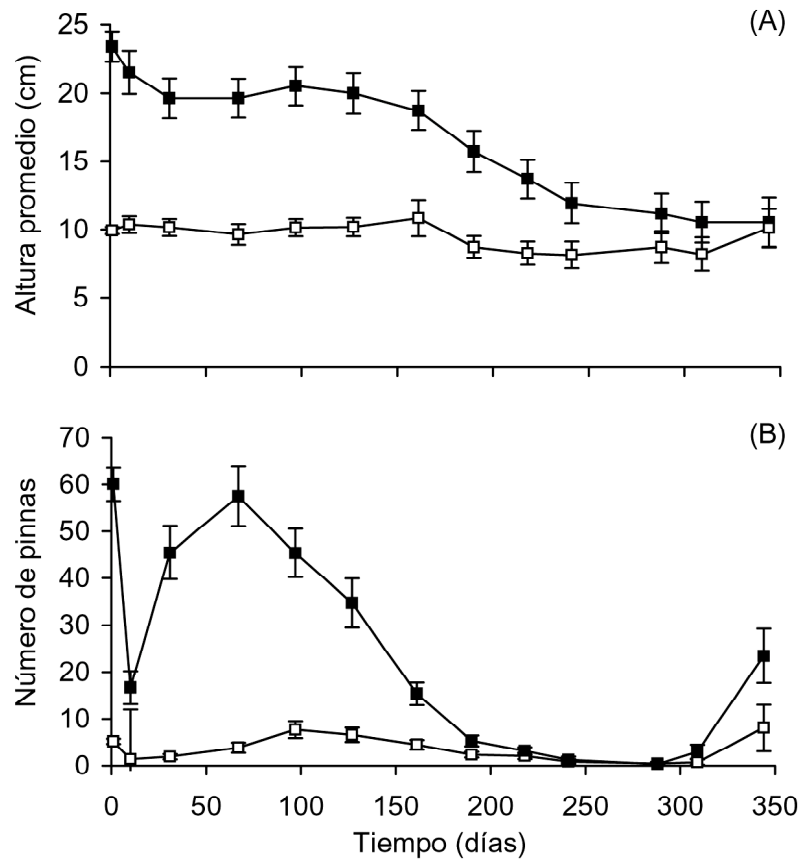

Figura 4. Altura promedio (A) y número promedio de pinnas por individuos (B) de plantas de Prosopis laevigata micorrizadas (símbolos llenos) y no micorrizadas (símbolos vacíos). Las barras de error son \pm 1 error estándar.
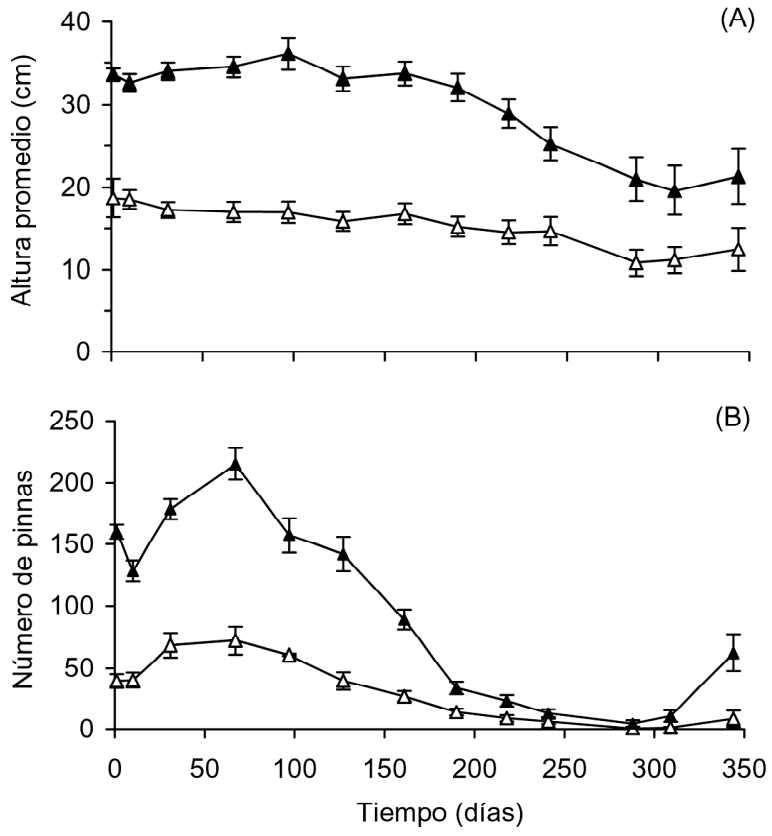

Figura 5. Altura promedio (A) y número promedio de pinnas por individuos (B), de plantas de Acacia farnesiana micorrizadas (símbolos llenos) y no micorrizadas (símbolos vacíos). Las barras de error son \pm 1 error estándar.

que tenían más esporas de los géneros Gigaspora y Scutellospora, promueven respuestas de crecimiento vegetal menores que Glomus. Así, es probable que el papel de inóculos con dominancia de Glomus sea promover el establecimiento y crecimiento de las plantas hospederas, mientras que las esporas más grandes de Gigaspora y Scutellospora promueven el desarrollo de la red hifal propia de ecosistemas intertropicales maduros (E. Allen, comentario personal).

El inóculo empleado favoreció el incremento en la altura de las plantas, registrado en los tratamientos micorrizados de las dos especies estudiadas (mezquite y huizache), durante el periodo de once meses de invernadero-vivero; al final de esta fase y al momento del trasplante en campo, el crecimiento fue de $c a$. el doble en las plantas micorrizadas respecto a sus testigos. En el caso del follaje de las dos especies, el número de pinnas fue de tres a seis veces superior en las plantas con HMA. Posteriomente, en el agostadero semiárido, la presión de herbivoría se ejerció de manera preferente sobre las plantas de mayor talla, lo que llevó a que las alturas de las plantas micorrizadas y no micorrizadas fueran similares al final del periodo de registro de datos; asimismo, el número de pinnas en ambas especies fue cercano a cero en los meses más secos (febrero-abril), debido a que son especies que desechan el follaje como mecanismo de resistencia a la sequía. Los resultados de cultivo de las dos especies en condiciones controladas (de invernadero o vivero), abren la posibilidad de desarrollar 
inóculos a base de HMA, para propagar especies nativas de zonas semiáridas con fines de restauración ecológica o con una finalidad productiva, como lo sugieren los trabajos de Siquiera et al. (1998), Bashan et al. (2000), Maestre et al. (2002b) y Caravaca et al. (2003).

Porcentaje de supervivencia. En relación con la pregunta inicial de este trabajo, se puede decir que la inoculación de plántulas de mezquite y huizache con HMA incrementa de manera significativa el porcentaje de supervivencia de plantas de las dos especies en condiciones de campo después de un ciclo anual, en el que los individuos deben sobrevivir a un largo periodo de sequía (octubre-abril) y a temperaturas extremas.

Estos resultados de incremento en la supervivencia de plantas formadoras de islas de recursos son relevantes para trabajos de recolonización vegetal con especies nativas en ecosistemas deteriorados, ya que se duplicó la supervivencia de las plantas en condiciones de campo y se mostró que la micorrización favorece la supervivencia de las plantas en el periodo más seco, debido posiblemente al suministro hídrico que aportan los HMA a su hospedero.

Islas de recursos. Es notable que la supervivencia de plantas de mezquite, micorrizadas o no micorrizadas, fue más elevada en caso de estar asociada a Karwinskia humbold tiana como planta nodriza, ya que esta especie no es apetecible para el ganado debido a su toxicidad (su nombre común es tullidora), por lo que ésta puede ser una especie nodriza que aminore la presión de herbivoría sobre plantas asociadas.

También es importante subrayar que la mortalidad de las plantas, tanto de mezquite como de huizache, se debió tanto a la herbivoría de conejos, liebres, ovejas y cabras, como al estrés hídrico causado por el periodo de sequía. Por ello, una planta no forrajeable como Karwinskia hum boldtiana es, posiblemente, una nodriza conveniente para el establecimiento de plantas, micorrizadas o no micorrizadas, cuando hay alta presión de herbivoría, como es el caso de los matorales xerófilos del Altiplano Mexicano. Asimismo, es recomendable evitar la presión de herbivoría debida a ganado doméstico (caprino y ovino) en parcelas experimentales, aunque en condiciones de campo existe una presión de herbivoría debida a animales silvestres (insectos, conejos, liebres, etc.), por lo que se requiere realizar experimentos con herbivoría controlada (cortando las hojas con tijeras, por ejemplo), si se desea conocer el reservorio de respuestas de plantas inoculadas con HMA, frente a la demanda de fotosintatos por parte del micobionte y ante la reducción del área fotosintética en el fitobionte, debida a la herbivoría (Gehring y Whitman, 1994; Wallig y Zabinski, 2006).

En este estudio no se encontraron diferencias estadísticas en la supervivencia de las plantas de mezquite y huizache atribuibles a la especie de nodriza o al tipo de isla de recursos; esto se debió a la alta variabilidad, en los datos de supervivencia de los individuos trasplantados, asociada a las diferentes especies de nodrizas empleadas y debida, probablemente, a la presión de herbivoría presente en un mat o ral xerófilo deteri o rado. Asimismo, se puede señalar que Opuntia cantabrigiensis no es una nodriza recomendable para favorecer el establecimiento de plantas de mezquite o huizache.

Establecimiento vegetal. La influencia de la inoculación con HMA sobre el desarrollo vegetativo de mezquite y huizache, en condiciones de campo, es más notable en el crecimiento del follaje, lo cual favorece el establecimiento vegetal. En efecto, el número de pinnas siempre fue superior en ambas especies en el caso de las plantas micorrizadas. Asimismo, las dos especies son caducifolias, perdiendo el follaje en la época más seca (febrero-abril); sin embargo, el rebrote de ramas y hojas es mayor en las plantas que fueron inoculadas con HMA. Estos resultados muestran la conveniencia de inocular plántulas de mezquite y huizache con HMA nativos, ya que son especies con relevancia ecológica y económica.

Finalmente es necesario señalar que en varios experimentos con plantas micorrizadas, sometidas a herbivoría, natural o simulada, no se ha llegado a conclusiones consistentes. Esto se debe a que intervienen factores como el tipo de inóculo, las especies de HMA involucradas, el tamaño y la fenología de la planta, la intensidad de la herbivoría y las condiciones edáficas, entre otros, por lo que no se puede generalizar el efecto de la herbivoría, sobre la respuesta de la planta y de los HMA simbiontes, para una misma especie vegetal, como es el caso del pasto norteamericano Bromus inermis Leysser (Klironomos, 2004). Por esta razón, en programas de restauración de comunidades vegetales deterioradas, es importante considerar la tasa de mortalidad debida al forrajeo por la fauna silvestre y el empleo de plantas no apetecibles o resistentes a la herbivoría.

\section{Agradecimientos}

Los autores agradecen los acertados comentarios de los revisores anónimos del primer manuscrito y las sugerencias de Jorge A. Meave, Editor en Jefe. Asimismo, se aprecia el trabajo de Leonardo Escalante García (que en paz descanse) por haber montado este experimento en condiciones de invernadero. También se agradece el apoyo financiero otorgado por la Dirección General de Asuntos del Personal Académico (DGAPA) de la Universidad Nacional Autónoma de México, mediante los proyectos IN-235402 e IN-213706, y en parte por un programa sectorial de CONACYT a través del proyecto con clave: SEMARNAT-2002C01-668. 


\section{Literatura citada}

Allen E.B., Allen M.F., Egerton-Warburton L., Cordiki L. y Gómez-Pompa A. 2003. Impacts of early-and late-seral mycorrhizae during restoration in seasonal tropical forest, Mexico. Ecological Applications 13:1701-1717.

Allen M.F. y Allen E.B. 1992. Development of mycorrhizal pat ches in a successional arid ecosystem. En: Read D. J., Lewis D.H., Fitter A.H. y Alexander I.J. Eds. Mycorrhizas in Ecosystems, pp.154-170, CAB Intemational, Oxon, Reino Unido.

Azcón-Aguilar C., Palenzuela J., Roldán A., Bautista S., Vallejo R. y Barea J.M. 2003. Analysis of the mycorrhizal potential in the rizhosphere of representative plant species from desertification-threatened Mediterranean shrublands. Applied Soil Ecology 22:29-37.

Azcón R. y Barea J.M. 1997. Mycorrhizal dependency of a representative plant species in Mediterranean shrublands (Lavandula spica L.) as key factor to its use for revegetation strategies in desertification-threatened areas. Applied Soil Ecology 7:83-92.

Barea J.M. 1998. Biología de la rizosfera. Investigación y Ciencia 47:74-81.

Bashan Y., Davis E.A., Carrillo-García A. y Linderman R.G. 2000. Assessment of VA mycorrhizal inoculum potential in relation to the establishment of cactus seedlings under mesquite nurse-trees in the Sonoran Desert. Applied Soil Ecology 14:165-175.

Callaway R. 1995. Positive interactions among plants. Botanical Review 61:306-349.

Camargo-Ricalde S.L. 2001. Some biological aspects of the arbuscular mycorrhizal fungi (AMF). Boletín de la Sociedad Botánica de México 68:15-32.

Camargo-Ricalde S.L. y Dhillion S.S. 2003. Endemic Mimosa species can serve as mycorrhizal "resource islands" within semiarid communities of the Tehuacán-Cuicatlán Valley, Mexico. Mycorrhiza 13:129-136.

Caravaca F., Barea J.M., Palenzuela J., Figueroa D., Alguacil M.M. y Roldán A. 2003. Establishment of shrub species in a degraded semiarid site after inoculation with native or allochtonous arbuscular mycorrhizal fungi. Applied Soil Ecology 22:103-111.

Challenger A. 1998. Utilización y Conservación de los Ecosistemas Terrestres de México. Pasado, Presente y Futuro. Comisión Nacional para el Conocimiento y Uso de la Biodiversidad, Universidad Nacional Autónoma de México y Agrupación Sierra Madre, S.C., México, D.F.

C ruz R.J.A. 1992. Interacciones entre los estratos arbóreo y arbustivo con la vegetación herbácea en una zona de matorral en el Valle de Actopan, Hidalgo. Tesis de Licenciatura (Biología), Escuela Nacional de Estudios Profesionales Zaragoza, Universidad Nacional Autónoma de México, México, D.F., 83 pp.

Cruz R.J.A. 1996. Evaluación de las condiciones microclimáticas, edáficas y de vegetación bajo el dosel de Prosopis laevigata (Humb. \& Bonpl. ex Willd.) M.C. Johnst., en un agostadero semiárido del norte de Guanajuato. Tesis de Maestría, Colegio de Postgraduados, Montecillo, Edo. de México, 100 pp.

Fagg C.W. y Stewart J.L. 1994. The value of Acacia and Prosopis in arid and semi-arid environments. Journal of Arid Environments 27:3-25.

Franco A.C. y Nobel P.S. 1989. Effect of nurse plants on the microhabitat and growth of cacti. Journal of Ecology 77:870886.

Galindo-Almanza S. y García-Moya E. 1986. The uses of mesquite (Prosopis spp.) in the highlands of San Luis Potosí, Mexico. Forest Ecology and Management 16:49-56.

García-Moya E. y McKell C.M. 1970. Contributions of shrubs to the nitrogen economy of a desert-wash plant community. Ecology 51:81-88.

Garner W. y Steinberger Y. 1989. A proposed mechanism for the formation of "fertile islands" in the desert ecosystems. Journal of Arid Environments 16:257-262.

Gehring C.A. y Whitman T.G. 1994. Interactions between aboveground herbivores and the mycorrhizal mutualist of plants. Trends in Ecology and Evolution 9:251-255.

Klironomos J.N., McCune J. y Moutoglis P. 2004. Species of arbuscular mycorrhizal fungi affect mycorrhizal responses to simulated herbivory. Applied Soil Ecology 26:133-141.

Maestre F.T., Bautista S., Cortina J., Díaz G., Honrubia M. y Vallejo V.R. 2002a. Microsite and mycomhizal inoculum effects on the establishment of Quercus coccifera in a semiarid degraded steppe. Ecological Engineering 19:289-295.

Maestre F.T., Huesca M.T., Zaady E., Bautista S. y Cortina J. 2002b. Infiltration, penetration resistance and microphytic crust composition in contrasted microsites within a Mediterranean semi-arid steppe. Soil Biology and Biochemistry 34:895-898.

Nobel P.S. 1998. Los Incomparables Agaves y Cactos. Trillas, México, D.F.

Rzedowski J. 1991. El endemismo en la fl ora fanerogámica mexicana: una apreciación analítica preliminar. Acta Botanica Mexicana 15:47-64.

Siqueira J.O., Carneiro M.A.C., Curi N., Da Silva Rosado S.C. y Davide A.C. 1998. Mycorrhizal colonization and mycotrophic growth of native woody species as related to successional groups in Southeastern Brazil. Forest Ecology and Management 107:241-252.

Valentin C., d'Herbes J.M. y Poesen J. 1999. Soil and water components of banded vegetation patterns. Catena 37:1-24.

Valiente-Banuet A. y Ezcurra E. 1991. Shade as a cause of the association between the cactus Neobuxbaumia tetetzo and the nurse plant Mimosa luisiana in the Tehuacán Valley, Mexico. Journal of Ecology 79:961-971.

Walling S.Z. y Zabinski C.A. 2006. Defoliation effects on arbuscular mycorrhizae and plant growth of two native bunchgrasses and an invasive forb. Applied Soil Ecology 32:111-117.

Wiegand K., Jeltsch F. y Ward D. 1999. Analysis of the population dynamics of Acacia trees in the Negev desert, Israel with a spatially-explicit computer simulation model. Ecological Modeling 117:2003-224.

Fecha de recepción: 21 de septiembre de 2005

Versión corregida: 6 de febrero de 2007

Aceptado: 20 de abril de 2007 